\title{
Tratamiento de pseudoaneurisma iatrogénico con compresión ecoguiada
}

\author{
Daniela González H. ${ }^{1}$, Sandra Hasbún A. ${ }^{1}$, Roberto Tapia R. ${ }^{1}$, Juan Carlos Herrera N. ${ }^{1}$, \\ Patricio Tepper L. ${ }^{1}$, Gabriela Molina G. ${ }^{1}$ y Gonzalo Contreras H. ${ }^{2}$
}

'Equipo Cirugía Vascular Hospital Regional de Talca, Servicio de Salud Maule, VII Región

${ }^{2}$ Universidad Católica del Maule, Chile.

Recibido el 11 de abril de 2017 y aceptado para publicación el 30 de junio de 2017

Correspondencia a: Daniela González H. danielad9@gmail.com

\section{Treatment of iatrogenic pseudoaneurysm with echo-guided compression}

Objective: To evaluate the effectiveness obtained with echo guided compression as the first choice to achieve thrombosis of iatrogenic pseudoaneurysm after study or interventionism. Material and Method: Retrospective observational study of 9 patients who underwent echo-guided compression as the first management alternative to iatrogenic pseudoaneurysms following diagnostic or therapeutic procedures that occurred between August 2012 and December 2015 at the Regional Hospital of Talca. Results: Of 4,070 procedures the complication was presented in 9 patients, $0.22 \% ; 7$ after therapeutic procedures $(0.4 \%)$, and 2 in the diagnostic group $(0.08 \%)$. The sample consists of 6 men and 3 women of average age 70 years. The involved vessel was mainly the femoral artery with $77 \%$. Pseudoaneurysms occurred mostly after therapeutic procedures (77\%); and the success of the echogenic compression was obtained in $67 \%$. There were no complications associated with treatment. Conclusions: Image guided compression has a lower success rate in this small series than those obtained by other authors, but since there are no complications and because of its low cost, we believe that it should be kept as the first alternative.

Key words: percutaneous access; pseudoaneurysms; image-guided compression.

\section{Resumen}

Objetivo: Evaluar la efectividad obtenida con la compresión ecoguiada como primera elección para lograr la trombosis del pseudoaneurisma iatrogénico post estudio o intervencionismo. Material y Método: Estudio retrospectivo observacional de 9 pacientes en quienes se realizó la compresión ecoguiada como primera alternativa de manejo frente a pseudoaneurismas iatrogénicos secundarios a procedimientos diagnósticos o terapéuticos ocurridos entre agosto de 2012 y diciembre de 2015 en el Hospital Regional de Talca. Resultados: De 4.070 procedimientos se presentó la complicación en 9 pacientes, un $0,22 \%$; 7 posterior a procedimientos terapéuticos $(0,4 \%)$, y 2 en el grupo de diagnósticos $(0,08 \%)$. La muestra se compone por 6 hombres y 3 mujeres de edad promedio 70 años. El vaso comprometido fue principalmente la arteria femoral con un $77 \%$. Los pseudoaneurismas ocurrieron mayoritariamente posteriores a procedimientos terapéuticos (77\%); y el éxito de la compresión ecoguiada se obtuvo en el $67 \%$. No hubo complicaciones asociadas al tratamiento. Conclusiones: La compresión ecoguiada tiene un porcentaje de éxito menor en esta pequeña serie que los obtenidos por otros autores, pero al no presentar complicaciones y por su bajo costo creemos debe mantenerse como primera alternativa.

Palabras clave: acceso percutáneo; pseudoaneurisma; compresión ecoguiada.

\section{Introducción}

El pseudoaneurisma es consecuencia de una disrupción de la pared arterial, ya sea por procesos inflamatorios, traumáticos o iatrogénicos. Esta última causal es la complicación más frecuente después del hematoma, como consecuencia a procedimientos endoluminales vasculares cardiacos o periféricos, y es comúnmente debida a compresión y hemostasia insuficiente después de una punción arterial. Su incidencia ha aumentado en las últimas décadas, con el desarrollo de las técnicas endovasculares, con valores que van de $0,2 \%$ a $0,5 \%$ en los procedimientos diagnósticos, y que llegan hasta un $7 \%$ cuando se 
asocian procedimientos terapéuticos, en relación a requerimientos de introductores de mayor diámetro, anticoagulación y antiagregación plaquetaria ${ }^{1-3}$.

Dependiendo del tamaño del pseudoaneurisma y presencia o no de sintomatología, su manejo puede variar desde la propuesta de una simple observación clínica, en el caso de aquellos menores de 20 a 30 $\mathrm{mm}$, hasta la cirugía tradicional, pasando por la compresión con o sin asistencia ultrasonográfica, inyección de trombina y otros procedimientos endovasculares.

Por muchos años el tratamiento de esta patología, cualquiera fuera su causa, fue la reparación quirúrgica del vaso afectado en aquellos de mayor tamaño, con un índice de complicaciones informadas no menores $^{4-6}$. Fellmeth, en 1991, presenta su experiencia de compresión ecoguiada con una casuística de 35 pacientes, teniendo un $70 \%$ de éxito, sin complicaciones ni recurrencia ${ }^{7}$. En otras revisiones se le atribuye a esta técnica una efectividad entre el $74 \%$ al $93 \%{ }^{8-10}$. El procedimiento es seguro, técnicamente sencillo y de bajo costo, principalmente como tratamiento de primera línea para lesiones recientes; ya que probablemente no es apropiado para las lesiones de larga data $^{7}$. La técnica tuvo excelente recepción y redujo drásticamente el número de pacientes sometidos a cirugía, disminuyendo el riesgo y la estadía hospitalaria de los enfermos.

Las contraindicaciones descritas según Fellmeth para este procedimiento incluyen la sospecha de infección, coexistencia de grandes hematomas con la eventual aparición de síndrome compartimental o isquemia, lesión de la piel que lo recubre, lesiones cercanas al ligamento inguinal, donde la ruptura podría ser catastrófica y el dolor intenso en la ingle que impida la compresión adecuada.

Actualmente, con el desarrollo de la cirugía mínimamente invasiva ha surgido otras alternativas terapéuticas como la inyección local de trombina (gold standard), la embolización con coils o el implante de stents recubiertos ${ }^{11,12}$, pero por simplicidad, bajo costo y buenos resultados creemos que el método descrito por Fellmeth continúa siendo una técnica de primera línea.

Se revisan y comunican los resultados de la técnica en los pacientes que nos ha tocado tratar en nuestro hospital.

\section{Material y Método}

Estudio retrospectivo observacional que contiene un reporte de una serie de casos con revisión de fichas clínicas de 9 pacientes en quienes se realizó la compresión ecoguiada en el Laboratorio de Cirugía Vascular como primera alternativa terapéutica frente al desarrollo de falso aneurisma poscateterismo en el Hospital Regional de Talca, entre agosto de 2012 y diciembre de 2015.

Los procedimientos fueron realizados por médicos del equipo o la enfermera de Cirugía Vascular quienes tienen horas designadas y hacen estudios en la Unidad en forma habitual. El equipo utilizado fue HD11XE marca Philips, con transductor C5-2.

Se incluyen todos los pacientes que presentaron esta complicación en nuestro Centro. Después de la sospecha clínica el diagnóstico de falso aneurisma fue confirmado con un dúplex.

Se recopilaron los siguientes datos para todos los pacientes incluidos: edad, sexo, comorbilidades, tiempo del diagnóstico e inicio del tratamiento después de la cateterización, si fue procedimiento diagnóstico o si existió terapia endovascular, el calibre de la vaina, el uso de antiplaquetarios y/o anticoagulantes.

La técnica utilizada fue, según lo descrito por Fellmeth, localización del cuello aneurismático y la compresión sobre él, ocluyendo el flujo a la cavidad, sin alterar el lumen del vaso. Este procedimiento dura 15 a 20 min, con control simultáneo de la cavidad hasta constatar la trombosis del pseudoaneurisma. Se repite el protocolo por 3 días o hasta lograr la trombosis, luego sello del sitio de compresión y reposo por $24 \mathrm{~h}$.

Se describen las características demográficas de los casos y éxito con la compresión ecoguiada.

\section{Resultados}

Durante el período estudiado se realizaron un total de 4.070 procedimientos presentando pseudoaneurisma 9 pacientes, lo que da una incidencia $0,22 \% ; 7$ posterior a procedimientos terapéuticos $(0,4 \%)$, y 2 en el grupo de diagnósticos $(0,08 \%)$.

En todos los pacientes el diagnóstico de falso aneurisma fue confirmado con ecografía dúplex, iniciando en ese momento el protocolo de compresión eco-guiada, obteniendo un éxito del 67\% (6 de los 9 pacientes del estudio) para lograr la efectiva trombosis del pseudoaneurisma en 1, 2 o 3 sesiones de compresión, de $20 \mathrm{~min}$ de duración cada una (Tabla 1). El procedimiento fue $100 \%$ tolerado por todos los pacientes.

El tiempo ocurrido entre el procedimiento y el diagnóstico de pseudoaneurisma varió de 1 hasta 19 días, como se muestra en la Tabla anterior.

La muestra se compone por 6 hombres y 3 muje- 
Tabla 1. Descripción de los pacientes según procedimiento, días hasta el diagnóstico, y si ésta tuvo éxito o no

\begin{tabular}{|lcccc|}
\hline & Procedimiento & $\begin{array}{c}\text { Días hasta el diagnóstico } \\
\text { de pseudoaneurisma }\end{array}$ & $\begin{array}{c}\text { Número de } \\
\text { compresiones }\end{array}$ & $\begin{array}{c}\text { Éxito con la compresión } \\
\text { ecoguiada }\end{array}$ \\
\hline Paciente 1 & Terapéutico & 4 & 3 & Sí \\
Paciente 2 & Terapéutico & 1 & 1 & No \\
\hline Paciente 3 & Diagnóstico & 7 & 1 & Sí \\
\hline Paciente 4 & Terapéutico & 8 & 1 & No \\
\hline Paciente 5 & Terapéutico & 1 & 2 & Sí \\
Paciente 6 & Diagnóstico & 14 & 3 & Sí \\
\hline Paciente 7 & Terapéutico & 1 & 1 & Sí́ \\
\hline Paciente 8 & Terapéutico & 11 & 3 & No \\
\hline Paciente 9 & Terapéutico & 19 & & Sí \\
\hline
\end{tabular}

Tabla 2. Comorbilidades

\begin{tabular}{|lll|}
\hline Patología & $\mathbf{n}$ & $\mathbf{\%}$ \\
\hline Hipertensión & 8 & 89 \\
\hline Diabetes & 3 & 33 \\
\hline Insuficiencia renal & 1 & 11 \\
\hline
\end{tabular}

res con un rango de edad que va desde los 59 a los 79 años, promedio 70 años. Las comorbilidades de éstos se describen en la Tabla 2.

El vaso comprometido fue principalmente la arteria femoral con un 77\% de los casos (Tabla 3) y los introductores utilizados fueron de 6 french en la mayoría (6 pacientes) y en el resto de 5 french.

Los pseudoaneurismas ocurrieron mayoritariamente posterior a procedimientos terapéuticos (78\%) y dentro de este grupo se encontraba el único paciente usuario de tratamiento anticoagulante oral. Además, de estos pacientes con causal intervencionismo terapéutico, sólo a 1 no se le administro terapia antiplaquetaria con clopidogrel posprocedimiento.

\section{Discusión}

No encontramos cifras nacionales en relación a incidencia de complicaciones del acceso vascular en intervencionismo y tampoco resultados con compresión ecoguiada.

La formación de pseudoaneurisma es la segunda complicación más común de los procedimientos endoluminales cardíacos y vasculares y su incidencia en nuestro grupo se encuentra en rango de lo espera-
Tabla 3. Lugar anatómico del pseudoaneurisma

\begin{tabular}{|lll|}
\hline Arteria & $\mathbf{n}$ & $\mathbf{\%}$ \\
\hline Femoral & 7 & 78 \\
\hline Humeral & 1 & 11 \\
\hline Radial & 1 & 11 \\
\hline
\end{tabular}

do según los resultados informados en la literatura ${ }^{1-3}$.

Nuestro porcentaje de éxito está bajo lo informado por Fellmeth y otras series ${ }^{7,13,14}$, pero sin existir complicaciones atribuidas al procedimiento consideramos estos aceptables en consideración a disponibilidad y bajo costo.

Dentro de las limitaciones del estudio está el hecho de que no tenemos registro del tamaño ni del cuello de los falsos aneurismas de todos nuestros pacientes, motivo por lo cual no se informa en esta revisión de casos, aunque no existe clara relación con éxito del tratamiento según esta variable ${ }^{14}$.

En nuestra serie, el diagnóstico del pseudoaneurisma fue secundario a sospecha clínica de este, y en vista de los buenos resultados publicados con la técnica, creemos que no justifican una conducta expectante, por el potencial riesgo de ruptura, teniendo en cuenta que son pacientes antiagregados generalmente. Aun así, creemos que pudiese existir en esta revisión un sesgo en la pesquisa y diagnóstico de pseudoaneurisma.

Por el pequeño volumen de pacientes no existe más información a destacar que el porcentaje de éxito del procedimiento en lograr la trombosis del pseudoaneurisma y la ausencia de complicaciones asociadas a la compresión ecoguiada. 


\section{Conclusión}

La compresión ecoguiada es una buena alternativa terapéutica como manejo de los pseudoaneurismas siendo un procedimiento sin complicaciones, de bajo costo, bien tolerada y con resultados aceptables y nuestra revisión así lo demuestra, a pesar de sus deficiencias.

\section{Responsabilidades éticas}

Protección de personas y animales. Los autores declaran que para esta investigación no se han reali- zado experimentos en seres humanos ni en animales.

Confidencialidad de los datos. Los autores declaran que en este artículo no aparecen datos de pacientes.

Derecho a la privacidad y consentimiento informado. Los autores declaran que en este artículo no aparecen datos de pacientes.

\section{Conflicto de intereses}

Los autores declaran que no tienen conflictos de interés.

\section{Bibliografía}

1. Katzenschlager R, Ugurluoglu A, Ahmadi A, Hülsmann M, Koppensteiner R, Larch $\mathrm{E}$, et al. Incidence of pseudoaneurysm after diagnostic and therapeutic angiography. Radiology 1995;195:463-6.

2. Kresowik T, Khoury M, Miller B, Winniford M, Shamma A, Sharp W, et al. A prospective study of the incidence and natural history of femoral vascular complications after percutaneous transluminal coronary angioplasty. J Vasc Surg. 1991;13:328-36.

3. Demirbas O, Batyraliev T, Eksi Z, Pershukov I. Femoral Pseudoaneurysm Due to Diagnostic or Interventional Angiographic Procedures. Angiology 2005;56:553-6.

4. San Norberto E, González J, Gutiérrez V, Carrera S, Vaquero C. Femoral pseudoaneurysms post-cardiac catheterization surgically treated: evolution and prognosis Interact CardioVasc Thorac Surg. 2009;3:353-8.

5. Dzijan-Horn M, Langwieser N, Groha P, Bradaric C, Linhardt M, Böttiger C, et al. Safety and Efficacy of a Potential Treatment Algorithm by Using Manual Compression Repair and Ultrasound-
Guided Thrombin Injection for the Management of Iatrogenic Femoral Artery Pseudoaneurysm in a Large Patient Cohort. Circ Cardiovasc Interv. 2014;7:207-15.

6. Lumsden AB, Miller JM, Kosinski AS, Allen RC, Dodson T F, Salam A A, et al A prospective evaluation of surgically treated groin complications following percutaneous cardiac procedures. Am Surg. 1994;60:132-7.

7. Fellmeth BD, Roberts AC, Bookstein JJ, Freischlag JA, Forsythe JR, Buckner NK, et al. Post-angiographic femoral artery injuries: nonsurgical repair with US-guided compression. Radiology 1991;178:671-5.

8. Briones J, Zaragoza J, Martínez C, Al-Raies B, Plaza A, Blanes J, et al. Tratamiento de los pseudoaneurismas iatrogénicos: comparación de la técnica de ecocompresión con la inyección ecoguiada de trombina humana. Rev Esp Angiol. 2006;58:445-50.

9. Schaub F, Theiss W, Busch R, Heinz M, Paschalidis M, Schomig A. Management of 219 consecutive cases of postcatheterization pseudoaneurysm. J Am Coll Cardiol. 1997;30:670-5.

10. Hernández I, Salgado J, Vidal JJ,
Segura RJ. Complicaciones vasculares postpunción de la arteria femoral en cateterismos cardiacos. Rev Esp Angiol. 2006;58:11-8.

11. Olsen D, Rodríguez J, Vranic M, Ramaiah V, Ravi R, Diethrich E, et al. Estudio prospectivo del tratamiento de los pseudoaneurismas de la arteria femoral con inyección de trombina guiada por ultrasonido: hacia una terapia menos invasiva. Angiología 2007;59:421-6.

12. Thalhammer C, Kirchherr AS, Uhlich F, Waigand J, Gross CM. Postcatheterization pseudoaneurysms and arteriovenous fistulas: repair with percutaneous implantation of endovascular covered stents. Radiology 2000;214:127-31.

13. Paulson E, Sheafor D, Kliewer M, Nelson R, Eisenberg L, Sebastian M, et al. Treatment of Iatrogenic Femoral Arterial Pseudoaneurysms: Comparison of US-guided Thrombin Injection with Compression Repair. Radiology 2000;215:403-8.

14. Cox G, Young J, Gray B, Grubb M, Hertzer N. Ultrasound-guided compression repair of postcatheterization pseudoaneurysms: Results of treatment in one hundred cases. J Vasc Surg. 1994;19:683-6. 